\title{
Statistical mechanics of money: How saving propensity affects its distribution
}

\author{
Anirban Chakraborti ${ }^{(1)}$ and Bikas K. Chakrabarti ${ }^{(2)}$ \\ Saha Institute of Nuclear Physics, \\ 1/AF Bidhan Nagar, Calcutta 700 064, India.
}

\begin{abstract}
We consider a simple model of a closed economic system where the total money is conserved and the number of economic agents is fixed. Analogous to statistical systems in equilibrium, money and the average money per economic agent are equivalent to energy and temperature, respectively. We investigate the effect of the saving propensity of the agents on the stationary or equilibrium probability distribution of money. When the agents do not save, the equilibrium money distribution becomes the usual Gibb's distribution, characteristic of non-interacting agents. However with saving, even for individual self-interest, the dynamics becomes cooperative and the resulting asymmetric Gaussian-like stationary distribution acquires global ordering properties. Intriguing singularities are observed in the stationary money distribution in the market, as functions of the marginal saving propensity of the agents.
\end{abstract}

PACS No. : 87.23.Ge, 05.90.+m, 89.90.+n, 02.50.-r 


\section{Introduction}

The interacting dynamical nature of any economic sector composed of many cooperatively interacting agents, has many features in common with the statistical physics of interacting systems. In fact, economists like Pareto investigated the power law properties of the wealth distributions more than a century ago and Stigler studied the market fluctuations by employing Monte Carlo methods more than thirty years back [1]. Motivated by these investigations, many efforts are being made recently to apply the statistical physics methods to various economic problems. A major part of the recent efforts has gone in investigating the nature of fluctuations and their distributions in the stock markets [2]. We believe, however, a fundamental dynamics occurs in the money market, which affects strongly the dynamics of other sectors in the economy. An understanding of the statistical mechanics of the money market is therefore essential, and some of its features are very intriguing [3, 4]. Dragulescu and Yakovenko [5] have shown very recently that for any arbitrary and random sharing but locally conserving money transaction between any two agents in a market, the money distribution goes to the equilibrium Gibb's distribution of statistical mechanics: $P(m)=(1 / T) \exp (-m / T)$ where $T=M / N$, the average money per agent in the market $(M$ is the total money of $N$ agents in the market). This equilibrium distribution is extremely robust and various kinds of inter-agent monetary transactions, which locally conserve the total money, lead to the Gibb's probability distribution satisfying $P\left(m_{1}\right) P\left(m_{2}\right)=P\left(m_{1}+m_{2}\right)$. A major achievement of this study [5] has been the precise identification of the temperature $T$ as the average money per agent in the market. This is due to the fact that the probability distribution is normalized and the total money is conserved. This precise identification had been missing in many of the previous attempts [3,4] though the identification from fiscal policy considerations was indeed very close [3]. They had considered a market consisting of $N$ agents, where initially each one gets any arbitrary share $m_{i}$ of the total money $M$ in the

market $\left(\sum_{i=1}^{N} m_{i}=M ; M\right.$ and $N$ fixed). The "trade" dynamics goes as follows. Select any two arbitrary agents $i$ and $j$ with money $m_{i}$ and $m_{j}$, respectively. These two agents then exchange their money through some trade, keeping their total amount of money $m_{i}+m_{j}$ conserved and no debt is allowed $\left(m_{i} \geq 0\right.$ at any stage of the trade). There is no other 
restriction in the trade. Extensive numerical simulations show that this and various modifications of trade, like multi-agent transactions, etc., all lead to the robust Gibb's distribution, independent of the initial distribution the market starts with. So, most of the agents end-up in this market with very little money! Supply of more money in the market (increasing T) can increase the width of the distribution, but the most probable money for any agent in the market remains zero. It may be mentioned that Ispolatov et al. had studied earlier the (non-equilibrium) wealth distributions in various asset exchange models [6] where the trade dynamics do not have time-reversal symmetry. Of course, the study of Levy and Solomon [7] indicates a power law distribution for the wealth in realistic (possibly non-equilibrium) markets or societies.

Although the model of Dragulescu and Yakovenko [5] is a simple and interesting one to start with, a very natural ingredient for any realistic economic agent is missing in the model: no economic agent trades with the entire money he or she possesses without saving a part of it; saving propensity is too natural a tendency for any economic agent [8]. This saving propensity of course varies from agent to agent and even with the accumulation of wealth of a single agent. There are also country-wise variations of this saving propensity. We note that the fraction of savings $\lambda$, called the "marginal propensity to save" by economists [8], remains fairly constant, independent of the agents. We have taken it to be a constant in the model considered here. We show that in presence of this saving factor $\lambda(\neq 0)$ the money market becomes truly cooperative in nature and "critical behaviour" [9] sets in. The multiplicative property of the Gibb's distribution $P(m)$, discussed above, was responsible for the absence of any cooperative feature of the statistics for $\lambda=0$. Once the local or individual measure of saving propensity is introduced $(\lambda \neq 0)$, a global order emerges in the entire money market,

giving a non-vanishing most probable money for each agent in the market and nontrivial critical behaviour of the resulting statistics in the equilibrium.

\section{Model and simulation results for money distribution}

We again consider a simple model of the closed economic system where the total amount of money $M$ is conserved and the number of economic agents $N$ is fixed. Each economic agent $i$, which may be an individual or a corporate, possesses money $m_{i}$. An economic agent 
can exchange money with any other agent through some trade, keeping the total amount of money of both the agents conserved. We assume that each economic agent saves a fraction $\lambda$ of its money $m_{i}$ before trading. We again assume that an agent's money must always be non-negative and therefore no debt is permitted. Let us now consider that an arbitrary pair of agents $i$ and $j$ get engaged in a trade so that their money $m_{i}$ and $m_{j}$ change by amounts $\Delta m_{i}$ and $\Delta m_{j}$ to become $m_{i}^{\prime}$ and $m_{j}^{\prime}$, where $\Delta m_{i}$ is a random fraction of $(1-\lambda)\left(m_{i}+m_{j}\right)$ and $\Delta m_{j}$ is the rest of it. Conservation of the total money in each trade is ensured, as earlier.

We performed computer simulations with fixed number of agents $N$ and total money $M=N T$. Most of our simulation results are for $N=500$ and $T=100$. However, we checked results for different $N$ values $(250 \leq N \leq 1000)$ to check finite size effects, etc., and with different $T$ values (upto 10000). Initially we divided the total money $M$ amongst $N$ agents equally so that $m_{i}=M / N=T$ for all $i$. We chose a fixed value of $\lambda$ between zero and unity and investigated its effects on the equilibrium distribution of money $P(m)$ in the market, giving the (normalized) number of agents $P$ with money $m$. We choose randomly two agents $i$ and $j$ having money $m_{i}$ and $m_{j}$, respectively. Then $\Delta m_{i}=\epsilon(1-\lambda)\left(m_{i}+m_{j}\right)$ and $\Delta m_{j}=(1-\epsilon)(1-\lambda)\left(m_{i}+m_{j}\right)$, where $\epsilon$ is a random number between zero and unity. Then $m_{i}^{\prime}=\lambda m_{i}+\Delta m_{i}$ and $m_{j}^{\prime}=\lambda m_{j}+\Delta m_{j}$ after the trade. Alternatively, this trade can also be viewed as $m_{i} \rightarrow m_{i}{ }^{\prime}, m_{j} \rightarrow m_{j}{ }^{\prime}$ where $m_{i}{ }^{\prime}=m_{i}-\Delta m, m_{j}{ }^{\prime}=m_{j}+\Delta m$ with $\Delta m=(1-\lambda)\left[m_{i}-\epsilon\left(m_{i}+m_{j}\right)\right]$. This can be checked by straightforward substitution. These trades were repeated for large number of arbitrary choices of pairs of agents; each of these random choices of pairs is considered as one trade and consequently one time unit. The typical time upto which we run this algorithm is above 5000 (sometimes upto 50000 for larger $\lambda$ ). The probability distribution was determined after every 50 time steps till a stationary distribution was obtained. We then took an average over 2000 such stationary distributions to obtain a smooth distribution. We checked that the equilibrium distribution obtained is again extremely robust and does not depend at all on the initial distribution of money in the market. We investigated the nature of the equilibrium distribution $P(m)$ for various values of $\lambda(0 \leq \lambda<1)$. Apart from the stationary distribution, we also investigated the "relaxation" 
behaviour [9] of the distributions and obtained the time variations of $P_{1} \equiv P(m=T)$ till a steady behaviour was found. We define the relaxation time $\tau_{R}$ as the earliest time where $P_{1}(t)$ becomes practically independent of time.

The results for the equilibrium distribution $P(m)$ are shown in Fig. 1, for some values of $\lambda$. The inset shows that the equilibrium distribution is independent of the market size $N$ and the average money in the market or temperature $T$. The real money exchanged randomly in any trade is less than the total money, because of the saving by each agent. This destroys the multiplicative property of the distribution $P(m)$ (seen earlier for $\lambda=0$ ) and $P(m)$ changes from the Gibb's form to the asymmetric Gaussian-like form as soon as a finite $\lambda$ is introduced. The $\lambda=0$ case was practically a random noise dominating one and therefore effectively a non-interacting market. Introduction of a finite amount of saving $(\lambda \neq 0)$, dictated by individual self-interest, immediately makes the money dynamics cooperative and the global ordering (in the distribution) is achieved. This kind of self-organization in the money market, coming out of pure self-interest of each agent, is reminiscent of the "invisible hand" effect $[8,10]$ in the "free market" suggested originally by Adam Smith in 1776.

The relaxation behaviour of the distribution is shown in Fig. 2, where the time variation of $P_{1}(t)$ for different values of $\lambda$ is shown. Since $m_{i} / T=1$ for all $i$ to start with, $P_{1}(t)$ starts falling from unity in all the cases (not shown in Fig. 2). After some initial rapid decay, we see an extremely slow spin-glass type $[4,9](\ln t)$ relaxation behaviour. The inset shows the typical variation of the relaxation time $\tau_{R}$ with $\lambda$. The dynamics obviously becomes slower with increasing $\lambda$ and $\tau_{R}$ seems to diverge as $\lambda$ approaches unity. Precisely at $\lambda=1$, the dynamics of course stops.

An important feature of this humped distribution $P(m)$ at any non-vanishing $\lambda$ is the variation of the most probable money $m_{p}(\lambda)$ ( where $P(m)$ becomes maximum) of the agents. As discussed before, $m_{p}=0$ for $\lambda=0$ (Gibb's distribution) and most of the economic agents in the market end-up losing most of their money. However, even with pure self-interest of each agent for saving a factor $\lambda$ of its own money at any trade, a global feature emerges: the entire market ends-up with a most-probable money $m_{p}(\lambda)$. This $m_{p}(\lambda)$ shifts in an interesting manner from $m_{p}=0$ (for $\lambda=0$ ) to $m_{p} \rightarrow T$ (for $\lambda \rightarrow 1$ ). We find that initially, 
for small $\lambda, m_{p}(\lambda)$ varies very closely as $\lambda^{1 / 2}$ and then it crosses over at $\lambda=\lambda_{c} \simeq 0.45$ to $\lambda^{1 / 3}$ variation. This is shown in Fig. 3, where the curves for $\lambda^{1 / 2}$ and $\lambda^{1 / 3}$ are also indicated. We do not have any idea why this crossover in the exponent for $\lambda$ occurs at any finite $\lambda(\simeq 0.45)$. Also the exponents $1 / 2$ and $1 / 3$ are very intriguing. We checked that this crossover point is perhaps also the point where the probability of an agent having zero money $P_{0} \equiv P(m=0)$ just disappears, as shown in the inset of Fig. 3. The half-width $\Delta m_{p}$ and the peak height $P_{m_{p}}$ of the equilibrium distribution, which scale practically as $(1-\lambda)^{1 / 2}$ and $(1-\lambda)^{-1 / 2}$ respectively (see Fig. 4), do not have any irregularity there. In fact, no other property of the equilibrium distribution $P(m)$ has any irregularity at $\lambda_{c}$. It may be mentioned that Ispolatov et al. also observed a singularity in the power law of non-equilibrium growth distribution of wealth in their multiplicative asset exchange model having broken time-reversal symmetric trade dynamics.

We noted that although the total distribution assumes some global cooperative feature, each individual's money $m_{i}$ fluctuates randomly. Fig. 5 shows the time variation of the money of an arbitrarily chosen individual in the market for two different values of $\lambda$. The inset shows the variation of the time-averaged money $\left\langle m_{i}\right\rangle$ of the agent and its fluctuation

$\Delta m_{i} \equiv \sqrt{\left\langle\left(m_{i}-\left\langle m_{i}\right\rangle\right)^{2}\right\rangle}$ with $\lambda$ after relaxation $\left(t>\tau_{R}\right)$. Since the total money is conserved, $\left\langle m_{i}\right\rangle$ remains constant $(=T)$ here, while $\Delta m_{i}$ goes down with $\lambda$ as $(1-\lambda)$. This is because at any time the agents keep a fixed fraction of their individual money and receive a random fraction of the money traded proportional to $(1-\lambda)$.

\section{Summary and discussion}

We thus considered here a very simple model of money market where the total money $M$ and the number of agents $N$ is fixed. Each pair of arbitrarily chosen agents in the market exchange money with each other through a trade (each trade considered as one time unit). During the trade, each agent saves a fraction $\lambda$ of its own money at that time and exchanges randomly out of the remaining money, conserving the total amount of money and not allowing any debt. We find that for $\lambda=0$, the market effectively becomes non-interacting and, no matter what initial distribution the agents start with, the resulting money distribution becomes the equilibrium Gibb's distribution [5], where most of the agents in the market end-up 
with very little money. For any non-vanishing $\lambda$, the equilibrium distribution becomes the asymmetric Gaussian-like with the most probable money $m_{p}$ (corresponding to the peak in $P(m))$ shifting away from $m=0$ with increasing $\lambda$. This global feature, coming out of the individual self-interest of saving a part of its own money, can be considered to be a demonstration of the self-organization in the market suggested long ago by Adam Smith ("invisible hand" $[8,10]$ ). Apart from this we find intriguing singularities appearing in the equilibrium distribution $P(m): m_{p} \sim \lambda^{\nu}$ where $\nu=1 / 2$ for $\lambda<\lambda_{c}(\simeq 0.45)$ and $\nu=1 / 3$ for $\lambda_{c}<\lambda<1$. Also, $\Delta m_{p} \sim(1-\lambda)^{1 / 2}$ and $P_{m_{p}} \sim(1-\lambda)^{-1 / 2}$.

These singularities in the equilibrium distribution come obviously from the cooperative nature of the market interactions induced by the saving propensity of the agents. It may be mentioned that while such singular behaviour in the equilibrium money distribution is very natural here, in the corresponding physical (gas) system of Newtonian particles one gets regular distributions, e.g., the Gibb's distribution (or for that matter, Bose or Fermi distributions for quantum particles) and never any singularity, because of the absence of any physical equivalent of the "saving" factor there. We thus believe, while regular distributions are common for minimally interacting physical many-body systems in equilibrium, singular distributions are typical of any working model of the markets.

Acknowledgements: We are grateful to A. Dutta, S. S. Manna, S. Pradhan and P. Sen for useful discussions, and S. Redner for some useful communications. We also thank the anonymous referee for suggesting important corrections and improvement of the manuscript. 


\section{References}

e-mail addresses :

(1)anirban@cmp.saha.ernet.in

(2)bikas@cmp.saha.ernet.in

1. V. Pareto, Cours d'Economie Politique, Lausanne and Paris (1897); G. J. Stigler, J. Business, 37, 117 (1964).

2. See e.g., R. N. Mantegna and H. E. Stanley, An Introduction to Econophysics, Cambridge University Press, Cambridge (2000).

3. B. K. Chakrabarti and S. Marjit, Ind. J. Phys., 69 B, 681 (1995).

4. S. Moss de Oliveira, P. M. C. de Oliveira and D. Stauffer, Evolution, Money, War and Computers, B. G. Teubner, Stuttgart (1999); P. W. Anderson, K. J. Arrow and D. Pines (Eds), The Economy as an Evolving Complex system, Addison-Wesley, Redwood City (1988).

5. A. Dragulescu and V. M. Yakovenko, cond-mat/0001439.

6. S. Ispolatov, P. L. Krapivsky and S. Redner, Euro. Phys. J. B, 2, 267-276 (1998).

7. M. Levy and S. Solomon, Physica A, 242, 90 (1997).

8. P. A. Samuelson, Economics, 11th Edition, McGraw-Hill Inc., Auckland, 195-208 (1980). 9. M. Plischke and B. Bergersen, Equilibrium Statistical Physics, 2nd Edition, World Scientific, Singapore (1994); S. K. Ma, Statistical Mechanics, World Scientific, Singapore (1985). 10. See e.g., J. M. Keynes, The General Theory of Employment, Interest and Money, The Royal Economic Society, Macmillan Press, London (1973). 


\section{Figure captions}

Fig. 1 : The stationary money distribution $P(m)$ versus money $m$ for different saving propensity factor $\lambda(N=500, T=100)$. The inset shows the same for different $N$ and $T$ at two fixed values of $\lambda$. The superposition of data indicates the absence of finite size effects and the temperature independence.

Fig. 2 : Relaxation of the distribution: $P_{1}(t)$ versus the time $t$ (number of trades) for different $\lambda$. Because of extremely slow relaxation, we show in the $\ln t$ scale for the later stage only. The vertical arrows indicate the relaxation time $\tau_{R}$. The inset shows the typical variation of $\tau_{R}$ with $\lambda$; $\tau_{R}$ diverges as $\lambda \rightarrow 1$.

Fig. 3 : The variation of the most probable money $m_{p}$ of an agent (peak position of the equilibrium distribution $P(m)$ ) as a function of $\lambda$. The dotted and dashed curves correspond to $\lambda^{1 / 2}$ and $\lambda^{1 / 3}$ respectively. The crossover point $\left(\lambda_{c}\right)$ is indicated by the vertical arrow. The inset shows that the probability $P_{0}$ of agents with zero money also first disappears at almost the same point $\left(\lambda_{c}\right)$ indicated by the vertical arrow.

Fig. 4 : The variation of the distribution half-width $\Delta m_{p}$ as function of $\lambda$. The dashed curve corresponds to $\sqrt{2.0(1-\lambda)}$. The inset shows the peak height $P_{m_{p}}$ as function of $\lambda$. The dotted curve here corresponds to $1 / \sqrt{2.4(1-\lambda)}$.

Fig. 5 : Time variation of money of an individual agent $m_{i}$ for two different values of $\lambda$. The inset shows the variations of $\left\langle m_{i}\right\rangle$ and its fluctuations $\Delta m_{i}$ as functions of $\lambda$. 


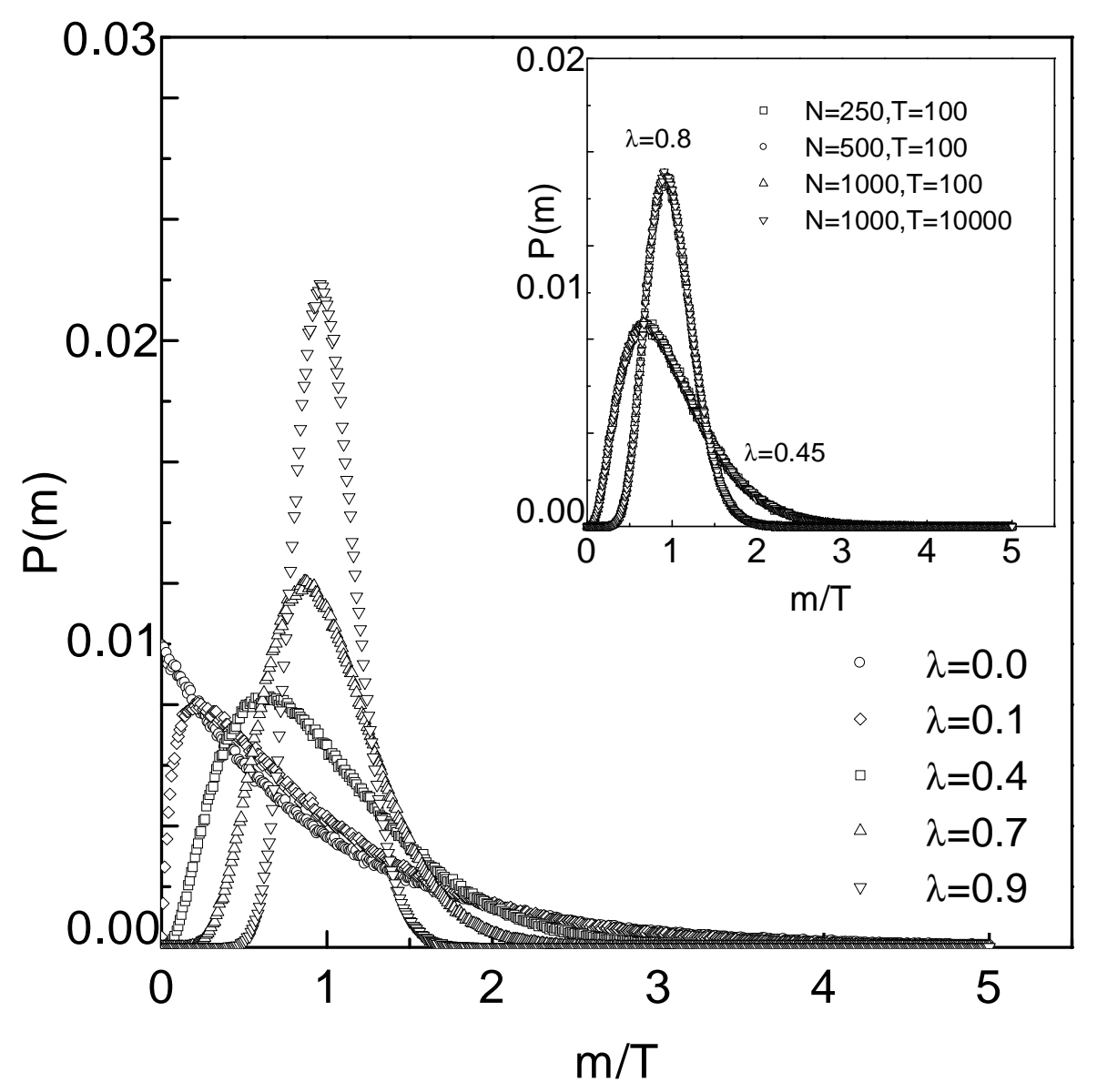

Fig. 1 


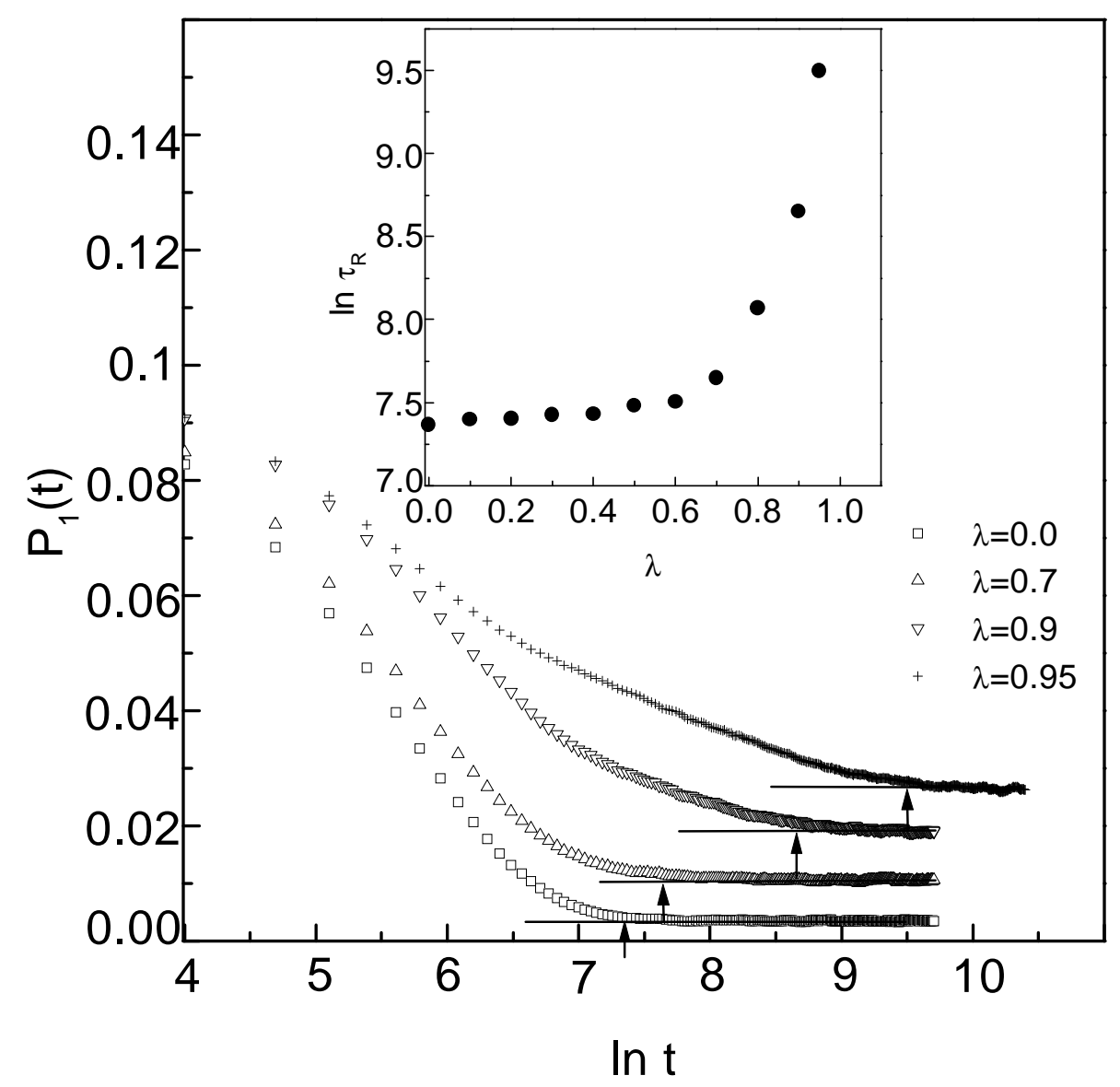

Fig. 2 


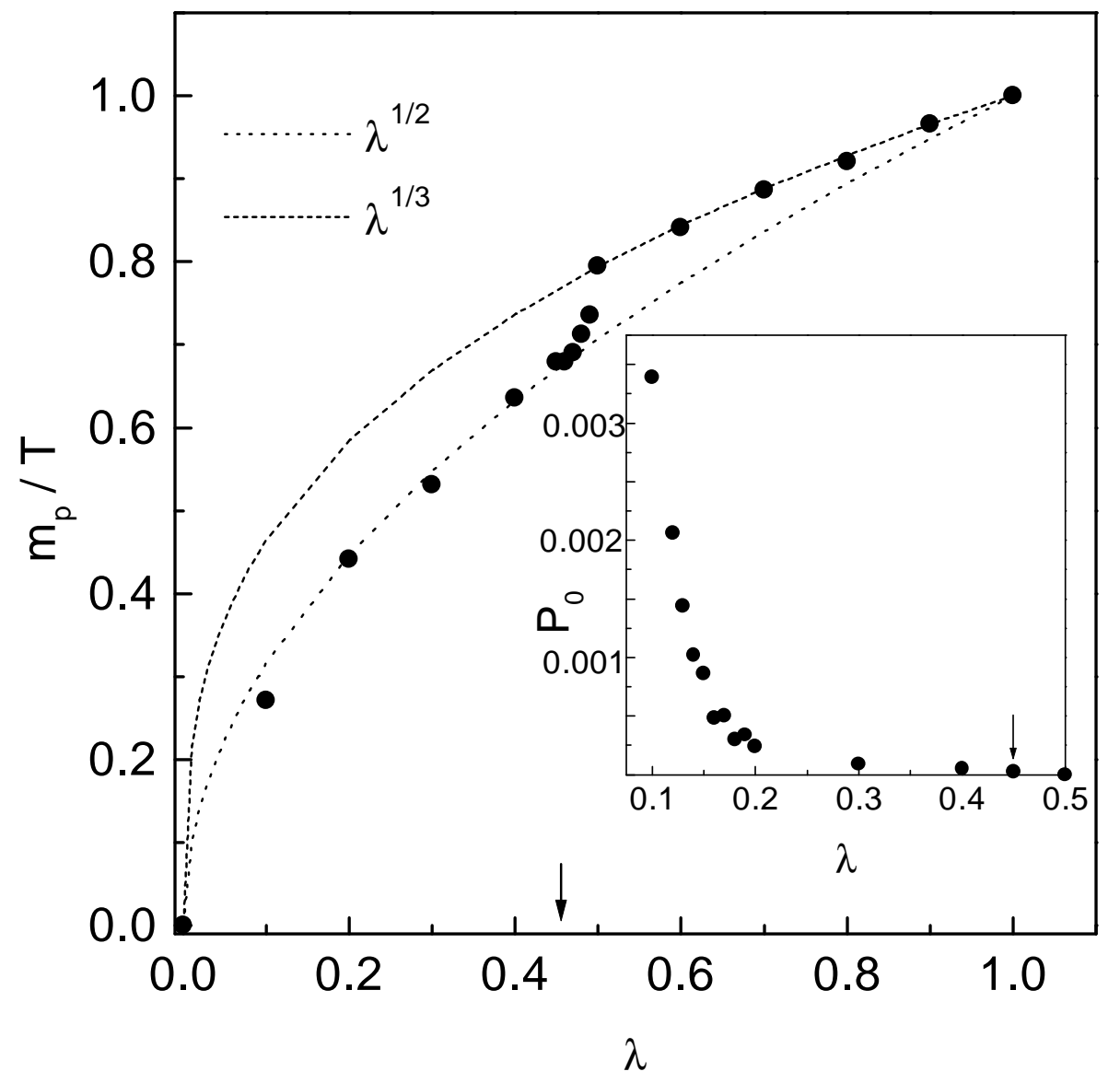

Fig. 3 


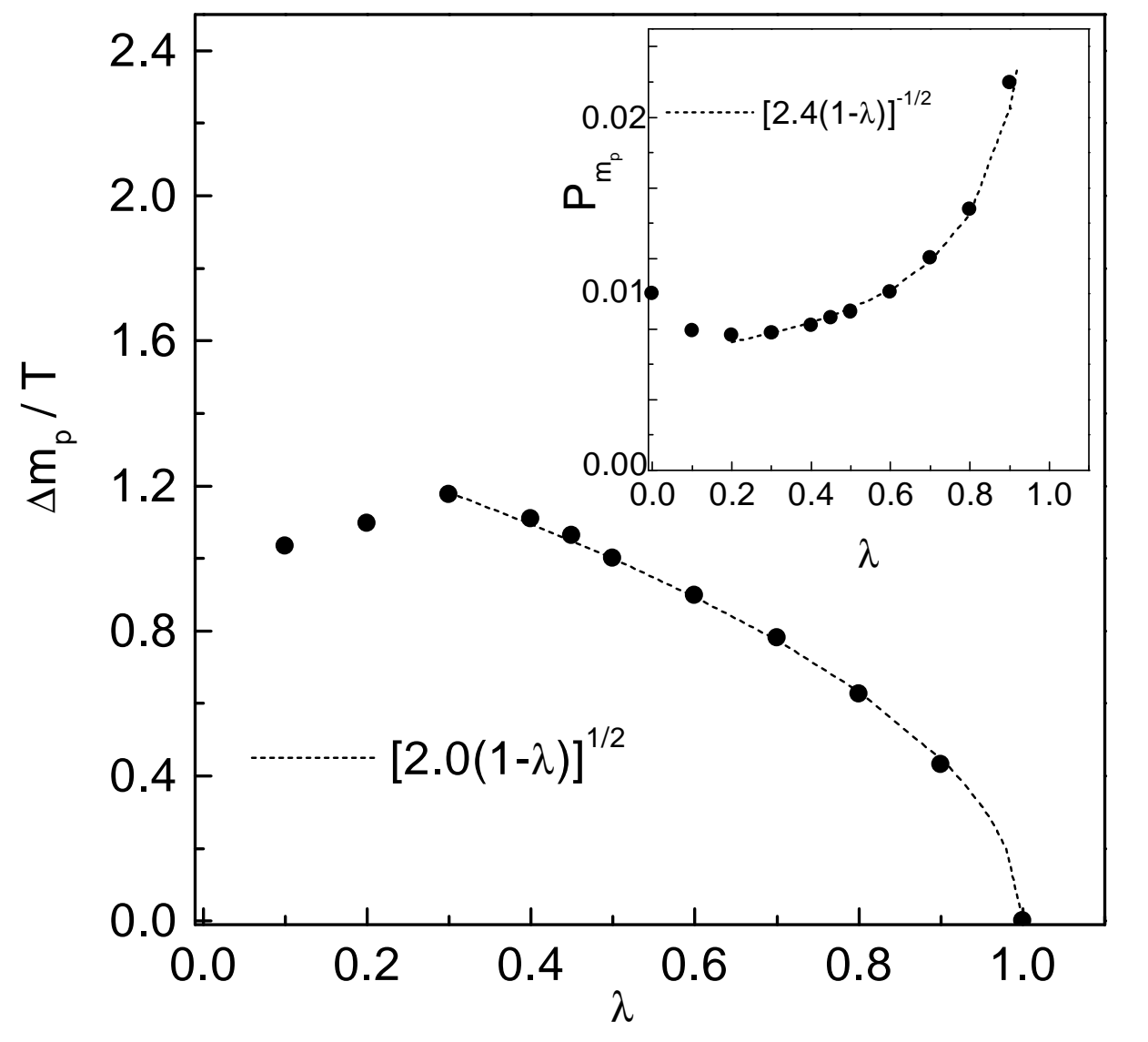

Fig. 4 


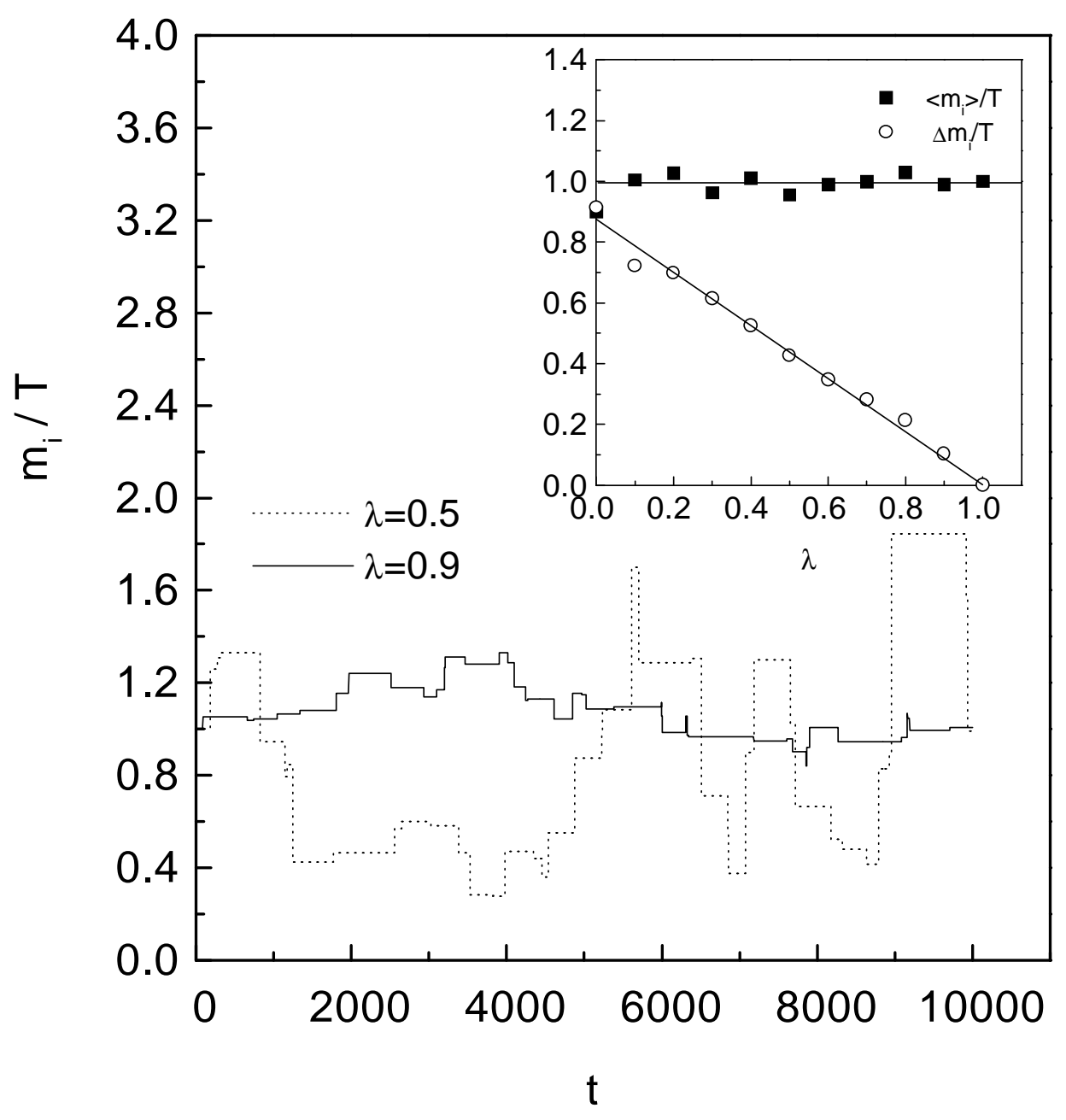

Fig. 5 